\title{
GEOMETRIC PROPERTIES OF SELF-SHRINKERS IN CYLINDER SHRINKING RICCI SOLITONS
}

\author{
MATHEUS VIEIRA AND DETANG ZHOU
}

\begin{abstract}
In this paper we prove some spectral properties of the drifted Laplacian of self-shrinkers properly immersed in gradient shrinking Ricci solitons. Then we use these results to prove some geometric properties of self-shrinkers. For example, we describe a collection of domains in the ambient space that cannot contain self-shrinkers.
\end{abstract}

\section{INTRODUCTION}

It is an important problem in geometry to study the singularities of the mean curvature flow. Self-shrinkers play a key role in the study of type I singularities of the flow. Recall that a self-shrinker is a submanifold of $\mathbb{R}^{n+1}$ satisfying

$$
\vec{H}+\frac{1}{2} x^{\perp}=0
$$

where $\vec{H}$ is the mean curvature vector and $x^{\perp}$ is the projection of the position vector to the normal bundle. Many important results in this topic have been done since the publication of Colding and Minicozzi [10].

Self-shrinkers are often compared with minimal surfaces. Hoffman and Meeks [17 proved that a nonplanar minimal surface properly immersed in $\mathbb{R}^{3}$ cannot lie in a open halfspace. In higher dimensions this result fails. The main difference is that a catenoid in $\mathbb{R}^{3}$ cannot lie in a open halfspace but a catenoid in $\mathbb{R}^{n+1}$ lies between two parallel hyperplanes for $n \geq 3$ (see [26] for properties of the higher dimensional catenoid). Recently, Cavalcante and Espinar [1] proved a halfspace theorem for self-shrinkers properly immersed in $\mathbb{R}^{n+1}$. The proof uses the idea of Hoffman and Meeks and a catenoid type self-shrinker discovered by Kleene and Moller [20. Note that there are many examples of compact self-shrinkers and noncompact proper self-shrinkers.

In this paper we show that strong halfspace type theorems for self-shrinkers are quite different from that of minimal surfaces and it is natural to use eigenvalues and eigenfunctions of the corresponding drifted Laplacian to prove them. We describe a collection of domains in the ambient space that cannot contain proper self-shrinkers. We also show some other geometric properties of self-shrinkers. Moreover, using spectral properties of the drifted Laplacian we can give short proofs of these results. Cheng and Zhou [7] proved that for a self-shrinker in $\mathbb{R}^{n+1}$ the conditions of proper immersion, Euclidean

The work was partially supported by CNPq and Faperj of Brazil. 
volume growth, polynomial volume growth and finite weighted volume are equivalent to each other. They also proved that the drifted Laplacian of a self-shrinker properly immersed in $\mathbb{R}^{n+1}$ has discrete spectrum [8]. Thus any eigenfunction of the drifted Laplacian with nonzero eigenvalue is orthogonal to the constant function with respect to the $L_{f}^{2}$ inner product. The spectrum of the drifted Laplacian contains the eigenvalues $\left\{0, \frac{1}{2}, 1\right\}$ with corresponding eigenfunctions $\left\{1 ; x_{1}, \ldots, x_{n+1} ; 2 n-|x|^{2}\right\}$. Applying the idea above to these eigenfunctions we easily prove the following result.

Theorem 1. Any self-shrinker hypersurface properly immersed in $\mathbb{R}^{n+1}$ intersects all members of the collection $\mathcal{C}$ given by

$$
\mathcal{C}:=\left\{S^{n}\left(t p, \sqrt{2 n+t^{2}}\right): p \text { is a unit vector in } \mathbb{R}^{n+1} \text { and } 0 \leq t \leq \infty\right\} .
$$

Moreover, if the self-shrinker lies in the closed set $\overline{B^{n+1}\left(t p, \sqrt{2 n+t^{2}}\right)}$, then either it is the sphere $S^{n}(o, \sqrt{2 n})$ with $t=0$, or it is the hyperplane $\langle x, p\rangle=$ 0 with $t=\infty$.

Remark 1. As $t \rightarrow \infty$ the sphere $S^{n}\left(t p, \sqrt{2 n+t^{2}}\right)$ converges to the hyperplane $\langle x, p\rangle=0$ and the closed ball $\overline{B^{n+1}\left(t p, \sqrt{2 n+t^{2}}\right)}$ converges to the closed halfspace $\langle x, p\rangle \geq 0$. For any vector $v$ in $\mathbb{R}^{n+1}$ the collection $\mathcal{C}$ contains the sphere $S^{n}\left(v, \sqrt{2 n+|v|^{2}}\right)$ as well the hyperplane $\langle x, v\rangle=0$.

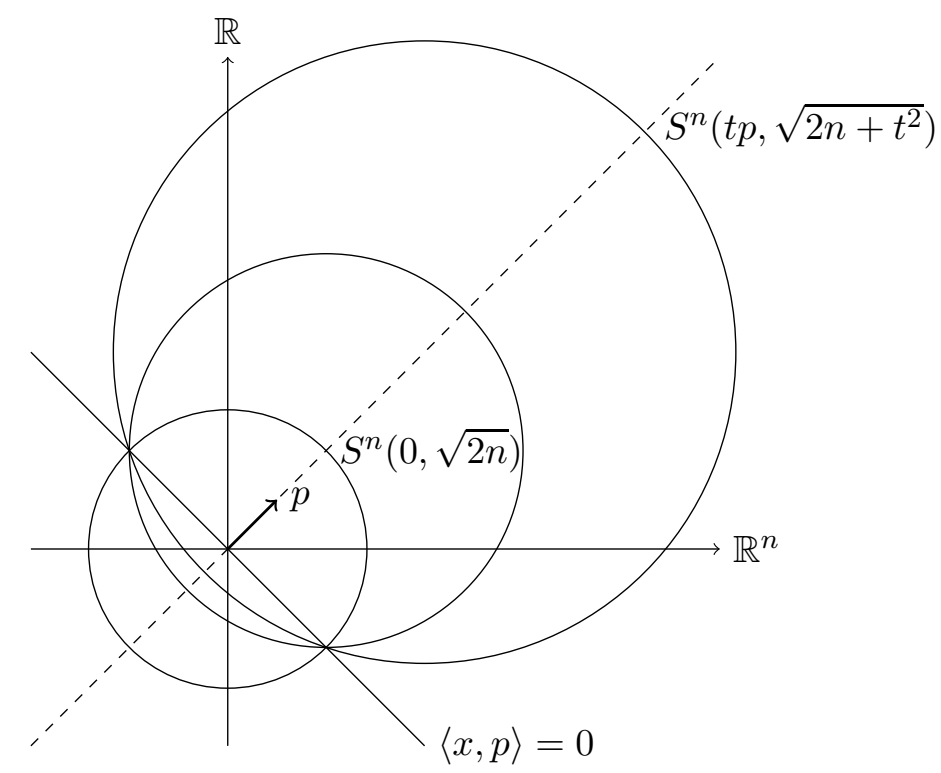

Among all self-shrinkers, spheres and cylinders deserve special attention since they are generic singularities of the mean curvature flow [10. Moreover, Colding and Minicozzi [11] proved that the blowup at each generic 
singularity of a mean curvature flow is unique; that is, it does not depend on the sequence of rescalings.

Extending Theorem 1 we prove the following result.

Corollary 1. Any self-shrinker hypersurface properly immersed in $\mathbb{R}^{n+1}$ satisfies the following geometric properties:

(a) If the Gaussian image of the self-shrinker lies in a closed semisphere, then it is a hyperplane.

(b) The self-shrinker cannot lie inside the closed product

$$
\overline{B^{k+1}\left(v, \sqrt{2 k+|v|^{2}}\right)} \times \mathbb{R}^{n-k}
$$

for any vector $v$ in $\mathbb{R}^{k+1}$, unless it is the product $S^{k}(o, \sqrt{2 k}) \times \mathbb{R}^{n-k}$ and $v=0$.

(c) The self-shrinker cannot lie outside the closed product

$$
\overline{B^{k+1}\left(v, \sqrt{2(k+1)+|v|^{2}}\right)} \times \mathbb{R}^{n-k}
$$

for any vector $v$ in $\mathbb{R}^{k+1}$.

(d) There is no hyperplane passing through the origin separating the selfshrinker into two parts with one part lying inside the closed ball $\overline{B^{n+1}(o, \sqrt{2 n})}$ and the other part lying outside the open ball $B^{n+1}(o, \sqrt{2 n})$, unless it is the sphere $S^{n}(o, \sqrt{2 n})$.

Remark 2. Item (a) was proved by Ding, Xin and Yang [12] by a different method.

It is also an interesting problem in geometry to study the mean curvature flow in more general ambient spaces. In the nonflat case Huisken [18] studied the asymptotic behavior of certain hypersurfaces evolving under the mean curvature flow immersed in the sphere. Recently, Sheng and Yu 25] generalized Huisken results to hypersurfaces evolving under the mean curvature flow immersed in Riemannian manifolds evolving under a normalized Ricci flow.

The study of the singularities of the mean curvature flow is related to $f$ minimal hypersurfaces and submanifolds. It is easy to see that a submanifold of $\mathbb{R}^{n+1}$ is a self-shrinker if and only if it is $f$-minimal for $f(x)=|x|^{2} / 4$. Recently, Yamamoto [28] proved that certain type I singularities of the Riccimean curvature flow are $f$-minimal submanifolds. More precisely, given a gradient shrinking Ricci soliton $(\bar{M}, g, f)$ and $T>0$ consider the canonical solution of the Ricci flow in the interval $-\infty<t<T$, and given a compact manifold $M$ consider a family of immersions $F: M \times[0, T) \rightarrow \bar{M}$ evolving under the mean curvature flow. Assuming that the coupled flow has a certain type I singularity at time $T$, Yamamoto proved that rescaled flow converges smoothly to a $f$-minimal submanifold of $\bar{M}$. Thus, a $f$-minimal submanifold 
of a gradient shrinking Ricci soliton appears as a singularity of the Riccimean curvature flow in the same way that a self-shrinker submanifold of $\mathbb{R}^{n+1}$ appears as a singularity of the mean curvature flow. In this paper $f$-minimal submanifolds of gradient shrinking Ricci solitons are also called self-shrinker submanifolds. Note that gradient shrinking Ricci solitons are also certain singularities of the Ricci flow (see [23] and the recent paper [14]).

Recently, Cheng, Mejia and Zhou extended the Choi-Wang estimate and the Colding-Minicozzi compactness theorem to $f$-minimal surfaces in [5] and [4] respectively. In [3] and [6] they classified index-one self-shrinker hypersurfaces properly immersed in the gradient shrinking Ricci soliton $\mathbb{R} \times$ $S^{n}(o, \sqrt{2(n-1)})$.

In this paper we focus in the case when the ambient space is a round cylinder gradient shrinking Ricci soliton $\left(\mathbb{R}^{p} \times S_{\sqrt{2(k-1)}}^{k}, g, f\right)$ with product metric $g$ and $f(x, y)=\frac{|x|^{2}}{4}$. Round cylinders are important in the study of the mean curvature flow. Colding and Minicozzi [10] proved that the generic singularities of the mean curvature flow are generalized round cylinders. They are also important in the study of the Ricci flow. Even though it is not known at this moment whether the generic singularities of the Ricci flow are generalized round cylinders, it is known that conformally flat gradient shrinking Ricci solitons are quotients of $S^{n}, \mathbb{R}^{n}$ and $\mathbb{R} \times S_{\sqrt{2(n-2)}}^{n-1}$. In fact, the works of Fernández-López and García-Río [15] and Munteanu-Sesum [22] led to the classification of gradient shrinking solitons of dimension $n \geq 4$ and harmonic Weyl tensor (vanishing Cotton tensor): they are either Einstein manifolds or a finite quotient of the product $\mathbb{R}^{n-k} \times N^{k}$ of the Gaussian shrinking soliton $\mathbb{R}^{n-k}$ with a positive Einstein manifold $N^{k}$ where $2 \leq k<$ $n$. We would like to point out that some results of this paper holds for more general gradient Ricci solitons. For example, the first part of Theorem 2 holds when the $S^{k}$ factor is replaced by any closed Einstein manifold with Einstein constant $\frac{1}{2}$ (see Lemma 4 for a even more general result).

The idea of using the spectral properties of the drifted Laplacian can be applied to submanifolds of gradient shrinking Ricci solitons. Cheng, Mejia and Zhou [4] proved that for a self-shrinker submanifold of $\mathbb{R}^{p} \times S_{\sqrt{2(k-1)}}^{k}$ the conditions of proper immersion, polynomial volume growth and finite weighted volume are equivalent to each other. By this fact, Lemma 3 and Lemma 4 we have the following result.

Theorem 2. Given a self-shrinker submanifold properly immersed in the gradient shrinking Ricci soliton $\mathbb{R}^{p} \times S_{\sqrt{2(k-1)}}^{k}$, the drifted Laplacian of the self-shrinker has discrete spectrum. Moreover, the spectrum contains the eigenvalues 0 and $\frac{1}{2}$ with corresponding eigenfunctions 1 and $x_{1}, \cdots, x_{p}$.

Remark 3. For $k=0$ the corresponding result for self-shrinker submanifolds properly immersed in $\mathbb{R}^{p}$ was proved in [8]. The proof uses a logarithmic 
Sobolev inequality obtained by Ecker [13. This inequality holds when the ambient space is a gradient shrinking Ricci soliton with bounded geometry 27. Here we give an alternative proof of the result in [8].

Applying spectral properties of the drifted Laplacian we prove the following result.

Theorem 3. Any self-shrinker hypersurface properly immersed in the gradient shrinking Ricci soliton $\mathbb{R}^{n+1-k} \times S_{\sqrt{2(k-1)}}^{k}$ satisfies the following geometric properties:

(a) If the self-shrinker lies inside the closed product $\overline{B_{\sqrt{2(n-k)}}^{n+1-k}} \times S_{\sqrt{2(k-1)}}^{k}$, then it is the product $S_{\sqrt{2(n-k)}}^{n-k} \times S_{\sqrt{2(k-1)}}^{k}$.

(b) The self-shrinker cannot lie outside the closed product $\overline{B_{\sqrt{2(n+1-k)}}^{n+1-k}} \times$ $S^{k} \sqrt{2(k-1)}$.

(c) If the self-shrinker lies in the product of a closed halfspace in $\mathbb{R}^{n+1-k}$ passing through the origin with $S_{\sqrt{2(k-1)}}^{k}$, then it is the product of the separating hyperplane with $S_{\sqrt{2(k-1)}}^{k}$.

The paper is organized as follows. In Section 2 we present the basic conventions and notations. In Section 3 we prove some spectral properties of the drifted Laplacian. In Section 4 we prove Theorem 1 , Corollary 1 and Theorem 3 ,

\section{Conventions and notations}

In this section we recall some definitions and present the conventions and notations of the paper.

Recall that a smooth metric measure space is a triple $(M, g, f)$ of a Riemannian manifold $(M, g)$ and a smooth function $f$ on $M$. The weighted volume is the measure $e^{-f} d v o l$. The drifted Laplacian is defined by

$$
\Delta_{f}=\Delta-\langle\nabla f, \nabla \cdot\rangle .
$$

The $L_{f}^{2}(M)$ inner product of functions $u$ and $v$ on $M$ is defined by

$$
\langle u, v\rangle_{L_{f}^{2}(M)}=\int_{M} u v e^{-f} .
$$

It is well known that the drifted Laplacian is a densely defined self-adjoint operator in $L_{f}^{2}(M)$, i.e. for smooth functions $u$ and $v$ on $M$ with compact we have

$$
\int_{M} \Delta_{f} u \cdot v e^{-f}=-\int_{M}\langle\nabla u, \nabla v\rangle e^{-f} .
$$

The Bakry-Émery-Ricci curvature is defined by

$$
\operatorname{Ric}_{f}=\operatorname{Ric}+\nabla \nabla f .
$$


A gradient Ricci soliton is a smooth metric measure space with

$$
\operatorname{Ric}_{f}=c g
$$

for some constant $c$. It is shrinking, steady or expanding if this constant is positive, zero or negative respectively.

Recall that for a submanifold $M$ of a smooth metric measure space $(\bar{M}, g, f)$ the second fundamental form is defined by

$$
A(X, Y)=\left(\bar{\nabla}_{X} Y\right)^{\perp} .
$$

Here we consider the Riemannian connection $\bar{\nabla}$ of the ambient space $\bar{M}$ and the projection $\perp$ to the normal bundle. The mean curvature vector is defined by

$$
\vec{H}=\operatorname{tr} A \text {. }
$$

The submanifold is called $f$-minimal if

$$
\vec{H}+(\bar{\nabla} f)^{\perp}=0 .
$$

It is well known that $M$ is $f$-minimal if and only if it is a critical point of the weighted volume functional $\operatorname{vol}_{f}(M)=\int_{M} e^{-f}$ with respect to compactly supported normal variations (e.g. [4]). Note that $(M, g, f)$ is also a smooth metric measure space and drifted Laplacian of $M$ is given by

$$
\Delta_{f}=\Delta-\langle\nabla f, \nabla \cdot\rangle .
$$

Here we consider the Riemannian connection $\nabla$ and the Laplacian $\Delta$ of the submanifold $M$.

In this paper by a self-shrinker submanifold we mean a $f$-minimal submanifold of gradient shrinking Ricci soliton. We usually denote the geometric quantities of the ambient space with a bar over them. The open ball $B^{k+1}(v, r)$ in $\mathbb{R}^{k+1}$ of radius $r$ and center $v$ has boundary $S^{k}(v, r)$ and closure $\overline{B^{k+1}(v, r)}$. The notation of closure for balls should not be confused with the notation of the ambient space. Sometimes we write $B_{r}^{k+1}=B^{k+1}(o, r)$ and $S_{r}^{k}=S^{k}(o, r)$.

Example 1. Consider the ambient space $\mathbb{R}^{p}$ with canonical metric $g$ and the function $f(x)=\frac{|x|^{2}}{4}$. The triple $\left(\mathbb{R}^{p}, g, f\right)$ is a gradient shrinking Ricci soliton with Bakry-Émery-Ricci curvature

$$
\overline{\operatorname{Ric}}_{f}=\frac{1}{2} g .
$$

A submanifold $M$ of $\mathbb{R}^{p}$ is a self-shrinker if and only if

$$
\vec{H}+\frac{1}{2} x^{\perp}=0
$$

The drifted Laplacian of $M$ is given by

$$
\Delta_{f}=\Delta-\frac{1}{2}\langle x, \nabla \cdot\rangle \text {. }
$$


The operator $\Delta_{f}$ is equal to the operator $\mathcal{L}$ introduced by Colding and Minicozzi [10].

Example 2. Consider the ambient space $\mathbb{R}^{p} \times S_{\sqrt{2(k-1)}}^{k}$ with product metric $g$ and the function $f(x, y)=\frac{|x|^{2}}{4}$. Here $x$ is the position vector in $\mathbb{R}^{p}$ and $y$ is the position vector in $\mathbb{R}^{k+1}$. The triple $\left(\mathbb{R}^{p} \times S_{\sqrt{2(k-1)}}^{k}, g, f\right)$ is a gradient shrinking Ricci soliton with Bakry-Émery-Ricci curvature

$$
\begin{aligned}
\overline{\operatorname{Ric}}_{f} & =\overline{\nabla \nabla} f+\overline{\operatorname{Ric}} \\
& =\frac{1}{2} g_{\mathbb{R}^{p}}+\frac{1}{2} g_{S_{\sqrt{2(k-1)}}} \\
& =\frac{1}{2} g .
\end{aligned}
$$

A submanifold $M$ of $\mathbb{R}^{p} \times S_{\sqrt{2(k-1)}}^{k}$ is a self-shrinker if and only if

$$
\vec{H}+\frac{1}{2} x^{\perp}=0
$$

The drifted Laplacian of $M$ is given by

$$
\Delta_{f}=\Delta-\frac{1}{2}\langle x, \nabla \cdot\rangle .
$$

\section{SpeCtral PRoperties of the DRIFted Laplacian}

In this section, first we characterize self-shrinker submanifolds of the gradient shrinking Ricci soliton $\mathbb{R}^{p} \times S_{\sqrt{2(k-1)}}^{k}$. In particular, for $k=0$ a submanifold of $\mathbb{R}^{p}$ is a self-shrinker if and only if the restrictions of the cartesian coordinates of $\mathbb{R}^{p}$ are eigenfunctions of the drifted Laplacian of the submanifold with eigenvalue $\frac{1}{2}$. Then we prove some differential equations and differential inequalities for self-shrinker submanifolds properly immersed in $\mathbb{R}^{p} \times S_{\sqrt{2(k-1)}}^{k}$. These identities and inequalities are used in the proofs of the results of next section. Then we prove that for self-shrinker submanifolds properly immersed in certain gradient shrinking Ricci solitons the spectrum of the drifted Laplacian of the submanifold is discrete. This result can be applied to self-shrinker submanifolds properly immersed in $\mathbb{R}^{p} \times S_{\sqrt{2(k-1)}}$. Finally, we prove a useful lemma for the drifted Laplacian of $f$-parabolic smooth metric measure spaces.

Lemma 1. For a complete self-shrinker submanifold $M$ of the gradient shrinking Ricci soliton $\mathbb{R}^{p} \times S_{\sqrt{2(k-1)}}^{k}$ the conditions proper immersion, polynomial volume growth and finite weighted volume (i.e. $\int_{M} e^{-f}<\infty$ ) are equivalent to each other. 
Proof. The conclusion follows from Corollary 1 in [4] because $\mathbb{R}^{p} \times S_{\sqrt{2(k-1)}}^{k}$ is a gradient shrinking Ricci soliton and the function $f(x, y)=\frac{|x|^{2}}{4}$ is convex.

Lemma 2. A submanifold $M$ of the gradient shrinking Ricci soliton $\mathbb{R}^{p} \times$ $S_{\sqrt{2(k-1)}}^{k}$ is a self-shrinker if and only if the cartesian coordinates $x_{1}, \ldots, x_{p}$ of $\mathbb{R}^{p}$ are eigenfunctions of drifted Laplacian of $M$ with eigenvalue $\frac{1}{2}$ i.e.

$$
\Delta_{f} x_{i}=-\frac{1}{2} x_{i}
$$

and the cartesian coordinates $y_{1}, \ldots, y_{k+1}$ of $\mathbb{R}^{k+1}$ are eigenfunctions of drifted Laplacian of $M$ with eigenvalue $\frac{1}{2(k-1)} \operatorname{tr}_{M} g_{S_{\sqrt{2(k-1)}}}$ i.e.

$$
\Delta_{f} y_{i}=-\left(\frac{1}{2(k-1)} \operatorname{tr}_{M} g_{S_{\sqrt{2(k-1)}}^{k}}\right) y_{i} \text {. }
$$

Proof. By direct calculation for any function $u$ on $\mathbb{R}^{p} \times S_{\sqrt{2(k-1)}}^{k}$ we have

$$
\nabla \nabla u=\overline{\nabla \nabla} u+\langle\bar{\nabla} u, A(\cdot, \cdot)\rangle .
$$

Here we consider the Riemannian connection $\nabla$ of $M$, the Riemannian connection $\bar{\nabla}$ of $\mathbb{R}^{p} \times S_{\sqrt{2(k-1)}}^{k}$ and the second fundamental form $A$ of $M$ with respect to $\mathbb{R}^{p} \times S_{\sqrt{2(k-1)}}^{k}$. Thus

$$
\begin{aligned}
\Delta_{f} u & =\operatorname{tr}_{M} \overline{\nabla \nabla} u+\langle\bar{\nabla} u, \vec{H}\rangle-\langle\nabla f, \nabla u\rangle \\
& =\operatorname{tr}_{M} \overline{\nabla \nabla} u+\left\langle\bar{\nabla} u, \vec{H}+(\bar{\nabla} f)^{\perp}\right\rangle-\langle\bar{\nabla} f, \bar{\nabla} u\rangle .
\end{aligned}
$$

The function $f(x, y)=\frac{|x|^{2}}{4}$ satisfies

$$
\bar{\nabla} f=\frac{1}{2} x .
$$

By direct calculation for any function $u$ on $\mathbb{R}^{p} \times \mathbb{R}^{k+1}$ we have

$$
\bar{\nabla} \nabla u=\widetilde{\nabla} \widetilde{\nabla} u+\langle\widetilde{\nabla} u, \bar{A}(\cdot, \cdot)\rangle .
$$

Here we consider the Riemannian connection $\widetilde{\nabla}$ of $\mathbb{R}^{p} \times \mathbb{R}^{k+1}$ and the second fundamental form $\bar{A}$ of $\mathbb{R}^{p} \times S_{\sqrt{2(k-1)}}^{k}$ with respect to $\mathbb{R}^{p} \times \mathbb{R}^{k+1}$, which is given by

$$
\bar{A}=-\frac{1}{2(k-1)} g_{S_{\sqrt{2(k-1)}}^{k}}(\cdot, \cdot) y .
$$

Take the canonical basis $e_{1}, \ldots, e_{p+k+1}$ of $\mathbb{R}^{p} \times \mathbb{R}^{k+1}$. Since $\widetilde{\nabla} y_{i}=e_{p+i}$ and $\widetilde{\nabla} \widetilde{\nabla} y_{i}=0$, by the two identities above we have

$$
\overline{\nabla \nabla} y_{i}=-\frac{1}{2(k-1)} y_{i} g_{S_{\sqrt{2(k-1)}}^{k}} .
$$


First assume that $M$ is a self-shrinker. Since $\bar{\nabla} x_{i}=e_{i}, \bar{\nabla} \nabla x_{i}=0$ and $\left\langle\bar{\nabla} f, \bar{\nabla} x_{i}\right\rangle=\frac{1}{2} x_{i}$, by Equation (1) we have

$$
\Delta_{f} x_{i}=-\frac{1}{2} x_{i}
$$

Since $\bar{\nabla} y_{i}=\operatorname{proj}_{T S_{\sqrt{2(k-1)}}^{k}} e_{p+i}$ and $\left\langle\bar{\nabla} f, \bar{\nabla} y_{i}\right\rangle=0$, by Equation (11) and Equation (2) we have

$$
\Delta_{f} y_{i}=-\left(\frac{1}{2(k-1)} \operatorname{tr}_{M} g_{S_{\sqrt{2(k-1)}}^{k}}\right) y_{i} .
$$

Now assume that $x_{i}$ and $y_{i}$ are eigenfunctions of the drifted Laplacian of $M$. Since $\Delta_{f} x_{i}=-\frac{1}{2} x_{i}$, taking $u=x_{i}$ in Equation (11) we obtain

$$
\left\langle\vec{H}+(\bar{\nabla} f)^{\perp}, e_{i}\right\rangle=0 .
$$

Since this identity holds for $i=1, \ldots, p$ it follows that

$$
\operatorname{proj}_{\mathbb{R}^{p}}\left(\vec{H}+(\bar{\nabla} f)^{\perp}\right)=0 .
$$

Since

$$
\Delta_{f} y_{i}=-\left(\frac{1}{2(k-1)} \operatorname{tr}_{M} g_{S_{\sqrt{2(k-1)}}^{k}}\right) y_{i}
$$

taking $u=y_{i}$ in Equation (11) and using Equation (2) we obtain

$$
\left\langle\vec{H}+(\bar{\nabla} f)^{\perp}, \operatorname{proj}_{T S_{\sqrt{2(k-1)}}} e_{p+i}\right\rangle=0 .
$$

Since this identity holds for $i=1, \ldots, k+1$ it follows that

Therefore

$$
\operatorname{proj}_{T S_{\sqrt{2(k-1)}}^{k}}\left(\vec{H}+(\bar{\nabla} f)^{\perp}\right)=0
$$

$$
\vec{H}+(\bar{\nabla} f)^{\perp}=0
$$

Corollary 2. A submanifold $M$ of $\mathbb{R}^{p}$ is a self-shrinker if and only if the cartesian coordinates $x_{1}, \ldots, x_{p}$ of $\mathbb{R}^{p}$ are eigenfunctions of the drifted Laplacian of $M$ with eigenvalue $\frac{1}{2}$.

Lemma 3. For a self-shrinker submanifold $M$ properly immersed in the gradient shrinking Ricci soliton $\mathbb{R}^{p} \times S_{\sqrt{2(k-1)}}^{k}$ the following assertions hold:

(a) $\Delta_{f}\langle x, v\rangle=-\frac{1}{2}\langle x, v\rangle$ for any $v$ in $\mathbb{R}^{p} \times \mathbb{R}^{k+1}$;

(b) $\Delta_{f}|x|^{2}=-|x|^{2}+2 p-2 \sum_{\alpha}\left|\operatorname{proj}_{\mathbb{R}^{p}} \nu_{\alpha}\right|^{2}$;

(c) $\Delta_{f}\left(\sum_{i=1}^{j} x_{i}^{2}\right) \geq-\sum_{i=1}^{j} x_{i}^{2}+2\left(j-\operatorname{dim} T M^{\perp}\right)$;

(d) $\Delta_{f}\left(\sum_{i=1}^{j} x_{i}^{2}\right) \leq-\sum_{i=1}^{j} x_{i}^{2}+2 j$.

Here $x$ is the position vector of $\mathbb{R}^{p}, x_{1}, \ldots, x_{p}$ are the cartesian coordinates of $\mathbb{R}^{p}$ and $\nu_{\alpha}$ is an orthonormal frame of the normal bundle of $M$. Moreover, the functions $\langle x, v\rangle,|x|^{2}$ and $\sum_{i=1}^{j} x_{i}^{2}$ and their gradients are in $L_{f}^{2}(M)$. 
Proof. First we prove Items (a), (b), (c) and (d). Item (a) follows from Lemma 2, We have

$$
\begin{aligned}
\Delta_{f}\left(\sum_{i=1}^{j} x_{i}^{2}\right) & =2 \sum_{i=1}^{j} \Delta_{f} x_{i} \cdot x_{i}+2 \sum_{i=1}^{j}\left|\nabla x_{i}\right|^{2} \\
& =-\sum_{i=1}^{j} x_{i}^{2}+2 \sum_{i=1}^{j}\left|\bar{\nabla} x_{i}\right|^{2}-2 \sum_{i=1}^{j}\left|\left(\bar{\nabla} x_{i}\right)^{\perp}\right|^{2} .
\end{aligned}
$$

Here we consider the Riemannian connections $\nabla$ and $\bar{\nabla}$ of $M$ and $\mathbb{R}^{p} \times$ $S_{\sqrt{2(k-1)}}^{k}$ respectively. On the other hand

$$
\begin{aligned}
\sum_{i=1}^{j}\left|\left(\bar{\nabla} x_{i}\right)^{\perp}\right|^{2} & =\sum_{i=1}^{j} \sum_{\alpha}\left\langle e_{i}, \nu_{\alpha}\right\rangle^{2} \\
& =\sum_{\alpha}\left|\operatorname{proj}_{\mathbb{R}^{j}} \nu_{\alpha}\right|^{2}
\end{aligned}
$$

where $e_{1}, \ldots, e_{j}$ is the canonical basis of $\mathbb{R}^{j}$. Thus

$$
\Delta_{f}\left(\sum_{i=1}^{j} x_{i}^{2}\right)=-\sum_{i=1}^{j} x_{i}^{2}+2 j-2 \sum_{\alpha}\left|\operatorname{proj}_{\mathbb{R}^{j}} \nu_{\alpha}\right|^{2} .
$$

This formula proves Items (b), (c) and (d).

To complete the proof it suffices to prove that the functions $\langle x, v\rangle$ and $\sum_{i=1}^{j} x_{i}^{2}$ and their gradients are in $L_{f}^{2}(M)$. Consider the open ball $B^{p}(o, r)$ in $\mathbb{R}^{p}$ of radius $r$ and center at the origin and the intrinsic open ball $B_{r}^{M}$ in $M$ of radius $r$ and center at a fixed point of $M$. Recall that $f(x, y)=\frac{|x|^{2}}{4}$. We have

$$
\begin{aligned}
\int_{M} f^{2} e^{-f} & \left.=\sum_{r=0}^{\infty} \int_{M \cap\left(\left(B^{p}(o, r+1) \backslash B^{p}(o, r)\right) \times S^{k} \sqrt{2(k-1)}\right.}\right) f^{2} e^{-f} \\
& \leq C_{1} \sum_{r=0}^{\infty}(r+1)^{4} e^{-\frac{r^{2}}{4}} \operatorname{vol}\left(M \cap\left(B^{p}(o, r+1) \times S_{\sqrt{2(k-1)}}^{k}\right)\right) \\
& \leq C_{2} \sum_{r=0}^{\infty}(r+1)^{4} e^{-\frac{r^{2}}{4}} \operatorname{vol}\left(B_{r+1}^{M}\right) \\
& \leq C_{3} \sum_{r=0}^{\infty}(r+1)^{4} e^{-\frac{r^{2}}{4}}(r+1)^{\alpha} \\
& <\infty
\end{aligned}
$$

where $\alpha>0$. In the third inequality above we used the fact that $M$ has polynomial volume growth (by Lemma 1). Thus

$$
\int_{M}|x|^{4} e^{-f}<\infty \text {. }
$$


Since $M$ has finite weighted volume (by Lemma 1) it follows that

$$
\begin{aligned}
\int_{M}\left(\sum_{i=1}^{j} x_{i}^{2}\right) e^{-f} & \leq\left(\int_{M}|x|^{4} e^{-f}\right)^{\frac{1}{2}}\left(\int_{M} e^{-f}\right)^{\frac{1}{2}} \\
& <\infty
\end{aligned}
$$

Clearly

$$
\begin{aligned}
\int_{M}\langle x, v\rangle^{2} e^{-f} & \leq|v|^{2} \int_{M}|x|^{2} e^{-f} \\
& <\infty \\
\int_{M}|\nabla\langle x, v\rangle|^{2} e^{-f} & \leq C_{4}|v|^{2} \int_{M} e^{-f} \\
& <\infty
\end{aligned}
$$

and

$$
\begin{aligned}
\int_{M}\left|\nabla \sum_{i=1}^{j} x_{i}^{2}\right|^{2} e^{-f} & \leq C_{5} \sum_{i=1}^{j} \int_{M}\left|x_{i}\right|^{2}\left|\nabla x_{i}\right|^{2} e^{-f} \\
& \leq C_{5} \sum_{i=1}^{j} \int_{M}\left|x_{i}\right|^{2} e^{-f} \\
& <\infty
\end{aligned}
$$

Lemma 4. For a self-shrinker submanifold $M$ properly immersed in a gradient shrinking Ricci soliton $(\bar{M}, g, f)$ with $f$ convex, the drifted Laplacian of $M$ with domain $L_{f}^{2}(M)$ has discrete spectrum.

Proof. Consider the Riemannian connections $\nabla$ and $\bar{\nabla}$ of $M$ and $\bar{M}$ respectively. We can assume that the Bakry-Émery-Ricci curvature of $\bar{M}$ satisfies $\overline{\operatorname{Ric}_{f}}=\frac{1}{2} g$. Consider the unitary isomorphism $U: L^{2}(M) \rightarrow L_{f}^{2}(M)$ given by $U u=u e^{\frac{f}{2}}$ and the operator $L=\Delta+\frac{1}{2} \Delta f-\frac{1}{4}|\nabla f|^{2}$. By direct calculation we have

$$
\Delta_{f}=U L U^{-1} \text {. }
$$

Thus the operator $\Delta_{f}$ with domain $L_{f}^{2}(M)$ has discrete spectrum if and only if the operator $L$ with domain $L^{2}(M)$ has discrete spectrum. By spectral theory to prove that $L$ has discrete spectrum it suffices to show that the function $\frac{1}{4}|\nabla f|^{2}-\frac{1}{2} \Delta f$ is proper (e.g. [24] page 120). Since

$$
\nabla \nabla f=\bar{\nabla} \nabla f+\langle\bar{\nabla} f, A(\cdot, \cdot)\rangle,
$$


it follows that

$$
\begin{aligned}
\frac{1}{4}|\nabla f|^{2}-\frac{1}{2} \Delta f & =\frac{1}{4}|\bar{\nabla} f|^{2}-\frac{1}{4}\left|(\bar{\nabla} f)^{\perp}\right|^{2}-\frac{1}{2} \operatorname{tr}_{M} \overline{\nabla \nabla} f-\frac{1}{2}\langle\bar{\nabla} f, \vec{H}\rangle \\
& =\frac{1}{4}|\bar{\nabla} f|^{2}+\frac{1}{4}|\vec{H}|^{2}-\frac{1}{2} \operatorname{tr}_{M} \overline{\nabla \nabla} f .
\end{aligned}
$$

This identity holds for any $f$-minimal submanifold of a smooth metric measure space. By [2] the scalar curvature of $\bar{M}$ satisfies $\bar{R} \geq 0$. By [16] $|\bar{\nabla} f|^{2}=f-\bar{R}$. Since $\bar{M}$ is gradient shrinking Ricci soliton it follows that $\bar{\Delta} f=\frac{\operatorname{dim} \bar{M}}{2}-\bar{R}$. Take a local orthonormal frame $\left\{\nu_{\alpha}\right\}$ in the normal bundle of $M$. We have

$$
\begin{aligned}
\frac{1}{4}|\nabla f|^{2}-\frac{1}{2} \Delta f & =\frac{1}{4}(f-\bar{R})+\frac{1}{4}|\vec{H}|^{2}-\frac{1}{2} \bar{\Delta} f+\frac{1}{2} \sum_{\alpha} \overline{\nabla \nabla} f\left(\nu_{\alpha}, \nu_{\alpha}\right) \\
& =\frac{1}{4}|\vec{H}|^{2}+\frac{f}{4}+\frac{\bar{R}}{4}-\frac{\operatorname{dim} \bar{M}}{4}+\frac{1}{2} \sum_{\alpha} \overline{\nabla \nabla} f\left(\nu_{\alpha}, \nu_{\alpha}\right) \\
& \geq \frac{f}{4}-\frac{\operatorname{dim} \bar{M}}{4} .
\end{aligned}
$$

Since $M$ is properly immersed in $\bar{M}$ and $f$ is a proper function on $\bar{M}$ (see [9]) it follows that the restriction of $f$ to $M$ is proper. This proves the result.

By the divergence theorem, for any smooth function $u$ on a compact smooth metric measure space $(M, g, f)$ we have $\int_{M} \Delta_{f} u \cdot e^{-f}=0$. But this is not generally true if $M$ is noncompact. It is an interesting problem to see when this formula holds because it has many geometric applications. We use the following lemma in the proofs of some results of the next section.

Lemma 5. Consider a complete smooth metric measure space $(M, g, f)$ with finite weighted volume, i.e. $\int_{M} e^{-f}<\infty$. Given a function $u$ on $M$ assume that $\nabla u$ is in $L_{f}^{2}(M)$. If the drifted Laplacian $\Delta_{f} u$ does not change sign or it is in $L_{f}^{1}(M)$, then

$$
\int_{M} \Delta_{f} u \cdot e^{-f}=0
$$

Proof. Consider the open ball $B_{r}$ of radius $r$ and center at a fixed point of $M$. Take a cutoff function $\phi$ on $M$ such that $\phi=1$ on $B_{r}, \phi=0$ on $M \backslash B_{r+1}, 0 \leq \phi \leq 1$ and $|\nabla \phi| \leq C$ on $B_{r+1} \backslash B_{r}$. Multiplying $\Delta_{f} u$ by $\phi$ and integrating by parts we obtain

$$
\int_{M} \Delta_{f} u \cdot \phi e^{-f}=-\int_{M}\langle\nabla u, \nabla \phi\rangle e^{-f} .
$$

By the monotone convergence theorem (if $\Delta_{f} u$ does not change sign) or the dominated convergence theorem (if $\Delta_{f} u$ is in $L_{f}^{1}(M)$ ), sending $r \rightarrow \infty$ we obtain

$$
\int_{M} \Delta_{f} u \cdot e^{-f}=-\lim _{r \rightarrow \infty} \int_{M}\langle\nabla u, \nabla \phi\rangle e^{-f}
$$


Since $M$ has finite weighted volume and $\nabla u$ is in $L_{f}^{2}(M)$ it follows from Holder's inequality that the limit on the right hand side is equal to zero.

The lemma above can be generalized to $f$-parabolic manifolds. The following result may be of independent interest.

Lemma 6. Suppose a $f$-parabolic complete smooth metric measure space $(M, g, f)$. Given a function $u$ on $M$ assume that $\nabla u$ is in $L_{f}^{2}(M)$. If the drifted Laplacian $\Delta_{f} u$ does not change sign or it is in $L_{f}^{1}(M)$, then

$$
\int_{M} \Delta_{f} u \cdot e^{-f}=0
$$

Proof. Take an exhausting sequence of open balls $\left\{B_{i}\right\}$ and a sequence of $f$-harmonic functions $\left\{\phi_{i}\right\}$ on $B_{i} \backslash B_{0}$ with boundary condition $\phi=1$ on $\partial B_{0}$ and $\phi=0$ on $B_{i}$. By the maximum principle we have

$$
\phi_{i} \leq \phi_{i+1}
$$

and by the divergence theorem we have

$$
\int_{B_{i} \backslash B_{0}}\left|\nabla \phi_{i}\right|^{2} e^{-f}=-\int_{\partial B_{0}} \frac{\partial \phi_{i}}{\partial \nu} e^{-f}
$$

Since $M$ is $f$-parabolic it follows that $\phi_{i} \rightarrow 1$ pointwise and

$$
\lim _{i \rightarrow \infty} \int_{B_{i} \backslash B_{0}}\left|\nabla \phi_{i}\right|^{2} e^{-f}=0,
$$

(e.g. [21] chapter 20). Extend $\phi_{i}=1$ in $B_{0}$ and $\phi_{i}=0$ in $M \backslash B_{i}$. Multiplying $\Delta_{f} u$ by $\phi_{i}$ and integrating by parts we obtain

$$
\int_{M} \Delta_{f} u \cdot \phi_{i} e^{-f}=-\int_{M}\left\langle\nabla u, \nabla \phi_{i}\right\rangle e^{-f}
$$

By the monotone convergence theorem (if $\Delta_{f} u$ does not change sign) or the dominated convergence theorem (if $\Delta_{f} u$ is in $L_{f}^{1}(M)$ ), sending $i \rightarrow \infty$ we obtain

$$
\int_{M} \Delta_{f} u \cdot e^{-f}=-\lim _{i \rightarrow \infty} \int_{M}\left\langle\nabla u, \nabla \phi_{i}\right\rangle e^{-f} .
$$

The limit on the right hand side is equal to zero since

$$
\left|\int_{M}\left\langle\nabla u, \nabla \phi_{i}\right\rangle e^{-f}\right| \leq\left(\int_{M}|\nabla u|^{2} e^{-f}\right)^{\frac{1}{2}}\left(\int_{M}\left|\nabla \phi_{i}\right|^{2} e^{-f}\right)^{\frac{1}{2}}
$$

and $\nabla u$ is in $L_{f}^{2}(M)$. 


\section{Proofs of Theorem 1 and Theorem 3}

We use the following lemma in the proofs of Theorem 1 and Corollary 1 .

Lemma 7. Given a vector $v$ in $\mathbb{R}^{k+1}$ and $r>0$ the hypersurface $S^{k}(v, r) \times$ $\mathbb{R}^{n-k}$ in $\mathbb{R}^{n+1}$ is a self-shrinker if and only if it is the product $S^{k}(o, \sqrt{2 k}) \times$ $\mathbb{R}^{n-k}$.

Proof. Take the canonical basis $e_{1}, \ldots, e_{k+1}$ and the cartesian coordinates $x_{1}, \ldots, x_{k+1}$ of $\mathbb{R}^{k+1}$. The hypersurface has normal vector $\nu=\frac{1}{r} \sum_{i=1}^{k+1}\left(x_{i}-v_{i}\right) e_{i}$ and mean curvature vector $\vec{H}=-\frac{k}{r} \nu$. Thus it is a self-shrinker if and only if

$$
\sum_{i=1}^{k+1} x_{i}\left(x_{i}-v_{i}\right)=2 k .
$$

In particular, $S^{k}(o, \sqrt{2 k}) \times \mathbb{R}^{n-k}$ is a self-shrinker.

Now assume that the hypersurface is a self-shrinker. Combining the equation above with

$$
\sum_{i=1}^{k+1}\left(x_{i}-v_{i}\right)^{2}=r^{2}
$$

we see that $S^{k}(v, r) \times \mathbb{R}^{n-k}=S^{k}(o, s) \times \mathbb{R}^{n-k}$ where $s^{2}=4 k-r^{2}+\sum_{i=1}^{k+1} v_{i}^{2}$. Thus $v=0$ and $r=\sqrt{2 k}$.

Now we prove Theorem 1 and Corollary 1 .

Proof of Theorem 1. The self-shrinker $M$ intercepts the sphere $S^{n}\left(t p, \sqrt{2 n+t^{2}}\right)$ for any unit vector $p$ in $\mathbb{R}^{n+1}$ and $0 \leq t<\infty$. To see this consider the function $u$ on $M$ given by

$$
u=2 n+t^{2}-|x-t p|^{2} .
$$

Suppose that $M$ does not intercept the sphere $S^{n}\left(t p, \sqrt{2 n+t^{2}}\right)$. Then $u$ is positive or negative. We have

$$
u=u_{1}+2 t u_{2}
$$

where $u_{1}=2 n-|x|^{2}$ and $u_{2}=\langle x, p\rangle$. By Lemma 3 the function $u$ is a linear combination of $L_{f}^{2}(M)$ eigenfunctions of the drifted Laplacian of $M$ with nonzero eigenvalues. Since $M$ has finite weighted volume (by Lemma 11) it follows that $u$ is orthogonal to the constant function with respect to the $L_{f}^{2}(M)$ inner product i.e. $\int_{M} u e^{-f}=0$, a contradiction.

The self-shrinker $M$ intercepts the halfspace $\langle x, p\rangle=0$ in $\mathbb{R}^{n+1}$ for any unit vector $p$ in $\mathbb{R}^{n+1}$. To see this consider the function $v$ on $M$ given by

$$
v=\langle x, p\rangle \text {. }
$$

Suppose that $M$ does not intercept the halfspace $\langle x, p\rangle=0$. Then $v$ is positive or negative. By Lemma 3 this function is a $L_{f}^{2}(M)$ eigenfunction of 
the drifted Laplacian of $M$ with nonzero eigenvalue. As before $\int_{M} v e^{-f}=0$, a contradiction.

By the facts above the self-shrinker $M$ intercepts all members of the collection $\mathcal{C}$.

Now assume that $M$ lies in the closed set $\overline{B^{n+1}\left(t p, \sqrt{2 n+t^{2}}\right)}$. For $0 \leq$ $t<\infty$ the function $u$ above is non-negative with $\int_{M} u e^{-f}=0$, so $u$ is identically zero and $M$ is the sphere $S^{n}\left(t p, \sqrt{2 n+t^{2}}\right)$, which by Lemma 7 is the sphere $S^{n}(o, \sqrt{2 n})$. For $t=\infty$ the function $v$ above is non-negative with $\int_{M} v e^{-f}=0$, so $v$ is identically zero and $M$ is the hyperplane $\langle x, p\rangle=0$.

Proof of Corollary 1. Assume that the Gaussian image of the self-shrinker $M$ lies in a closed semisphere. Given a vector $v$ in $\mathbb{R}^{n+1}$ consider the function $u$ on $M$ given by

$$
u=\langle\nu, v\rangle \text {. }
$$

Then $u$ is non-negative for some vector $v$ in $\mathbb{R}^{n+1}$. By Lemma 5.5 in [10] (or Proposition 2 in [3]) we have

$$
\Delta_{f} u+|A|^{2} u=0 .
$$

If $u$ is identically zero then $M$ is a hyperplane. If $u$ is not identically zero then the first eigenvalue of the operator $\Delta_{f}+|A|^{2}$ is non-negative, so

$$
\int_{M}|A|^{2} \phi^{2} e^{-f} \leq \int_{M}|\nabla \phi|^{2} e^{-f}
$$

for all smooth functions $\phi$ on $M$ with compact support. Consider the intrinsic open ball $B_{r}$ on $M$ of radius $r$ and center at a fixed point of $M$. Take a cutoff function $\phi$ on $M$ such that $\phi=1$ on $B_{r}, \phi=0$ on $M \backslash B_{r+1}$, $0 \leq \phi \leq 1$ and $|\nabla \phi| \leq C$ on $B_{r+1} \backslash B_{r}$. Since $M$ has finite weighted volume (by Lemma 11), by the monotone convergence theorem sending $r \rightarrow \infty$ we see that that $A$ is identically zero and $M$ is a hyperplane.

Suppose that the self-shrinker $M$ lies inside the closed product

$$
\overline{B^{k+1}\left(v, \sqrt{2 k+|v|^{2}}\right)} \times \mathbb{R}^{n-k} .
$$

Consider the function $u$ on $M$ given by

$$
u=\sum_{i=1}^{k+1}\left(x_{i}-v_{i}\right)^{2}-2 k-\sum_{i=1}^{k+1} v_{i}^{2} .
$$

Then $u$ is non-positive. We have

$$
u=u_{1}-2 u_{2},
$$

where $u_{1}=\sum_{i=1}^{k+1} x_{i}^{2}-2 k$ and $u_{2}=\sum_{i=1}^{k+1} x_{i} v_{i}$. By Lemma 3 the functions $u_{1}$ and $u_{2}$ and their gradients are in $L_{f}^{2}(M)$ and satisfy $\Delta_{f} u_{1} \geq-u_{1}$ and $\Delta_{f} u_{2}=-\frac{1}{2} u_{2}$. Thus

$$
0 \geq u \geq-\Delta_{f} u_{1}+4 \Delta_{f} u_{2}
$$


To prove that $u$ is identically zero it suffices to show that $\int_{M} \Delta_{f} u_{1} \cdot e^{-f}=0$ and $\int_{M} \Delta_{f} u_{2} \cdot e^{-f}=0$. The function $u_{2}$ is a $L_{f}^{2}(M)$ eigenfunction of the drifted Laplacian of $M$ with nonzero eigenvalue. Since $M$ has finite weighted volume (by Lemma 1) it follows that $u_{2}$ is orthogonal to the constant function with respect to the $L_{f}^{2}(M)$ inner product i.e. $\int_{M} u_{2} e^{-f}=0$, so $\int_{M} \Delta_{f} u_{2} \cdot e^{-f}=0$. By Lemma 3 we have

$$
\left|\Delta_{f} u_{1}\right| \leq \sum_{i=1}^{k+1} x_{i}^{2}+2(k+1) .
$$

By Lemma1 1 and Lemma 3 the functions in the right hand side are in $L_{f}^{1}(M)$. It follows from Lemma 5 that $\int_{M} \Delta_{f} u_{1} \cdot e^{-f}=0$. Thus $u$ is identically zero and $M$ is the product $S^{k}\left(v, \sqrt{|v|^{2}+2 k}\right) \times \mathbb{R}^{n-k}$, which by Lemma 7 is the product $S^{k}(o, \sqrt{2 k}) \times \mathbb{R}^{n-k}$.

Suppose that the self-shrinker $M$ lies outside the closed product

$$
\overline{B^{k+1}\left(v, \sqrt{|v|^{2}+2(k+1)}\right)} \times \mathbb{R}^{n-k} .
$$

Consider the function $u$ on $M$ given by

$$
u=\sum_{i=1}^{k+1}\left(x_{i}-v_{i}\right)^{2}-2(k+1)-\sum_{i=1}^{k+1} v_{i}^{2} .
$$

Then $u$ is non-negative. We have

$$
u=u_{1}-2 u_{2},
$$

where $u_{1}=\sum_{i=1}^{k+1} x_{i}^{2}-2(k+1)$ and $u_{2}=\sum_{i=1}^{k+1} x_{i} v_{i}$. By Lemma 3 the functions $u_{1}$ and $u_{2}$ and their gradients are in $L_{f}^{2}(M)$ and satisfy $\Delta_{f} u_{1} \leq$ $-u_{1}$ and $\Delta_{f} u_{2}=-\frac{1}{2} u_{2}$. Thus

$$
0 \leq u \leq-\Delta_{f} u_{1}+4 \Delta_{f} u_{2} .
$$

As before $u$ is identically zero and $M$ is the product $S^{k}\left(v, \sqrt{|v|^{2}+2(k+1)}\right) \times$ $\mathbb{R}^{n-k}$. By Lemma 7 this product is not a self-shrinker, a contradiction.

Suppose that there is a hyperplane $\langle x, v\rangle=0$ in $\mathbb{R}^{n+1}$ separating the self-shrinker $M$ into two parts with one part lying inside the closed ball $\overline{B^{n+1}(o, \sqrt{2 n})}$ and the other part lying outside the open ball $B^{n+1}(o, \sqrt{2 n})$. Consider the function $u$ on $M$ given by

$$
u=\left(2 n-|x|^{2}\right)\langle x, v\rangle .
$$

Then $u$ is non-negative or non-positive. We have

$$
u=u_{1} u_{2},
$$


where $u_{1}=2 n-|x|^{2}$ and $u_{2}=\langle x, v\rangle$. By Lemma 3 the functions $u_{1}$ and $u_{2}$ are $L_{f}^{2}(M)$ eigenfunctions of the drifted Laplacian of $M$ with distinct eigenvalues, so $u_{1}$ is orthogonal to $u_{2}$ with respect to the $L_{f}^{2}(M)$ inner product i.e. $\int_{M} u_{1} u_{2} e^{-f}=0$. Thus $u$ is identically zero and $M$ is the sphere $S^{n}(o, \sqrt{2 n})$.

By the proof of Lemma 7 we have the following result.

Lemma 8. Given $r>0$ the hypersurface $S_{r}^{n-k} \times S_{\sqrt{2(k-1)}}^{k}$ in the gradient shrinking Ricci soliton $\mathbb{R}^{n+1-k} \times S_{\sqrt{2(k-1)}}^{k}$ is a self-shrinker if and only if it is the product $S_{\sqrt{2(n-k)}}^{n-k} \times S_{\sqrt{2(k-1)}}^{k}$.

Now we prove Theorem 3 ,

Proof of Theorem 3. Suppose that the self-shrinker $M$ lies inside the closed product $\overline{B^{n+1-k}} \times S_{\sqrt{2(n-k)}}^{k}$. Consider the function $u$ on $M$ given by

$$
u=2(n-k)-|x|^{2},
$$

where $x$ is the position vector in $\mathbb{R}^{n+1-k}$. Then $u$ is non-negative. By Lemma 3 the function $u$ and its gradient are in $L_{f}^{2}(M)$ and satisfies

$$
0 \leq u \leq-\Delta_{f} u \text {. }
$$

Since $\Delta_{f} u$ does not change sign, by Lemma 5 we have $\int_{M} \Delta_{f} u \cdot e^{-f}=0$. Thus $u$ is identically zero and $M$ is the product $S_{\sqrt{2(n-k)}}^{n-k} \times S_{\sqrt{2(k-1)}}^{k}$.

Suppose that the self-shrinker $M$ lies outside the product $\frac{\sqrt{2(k-1)}}{B^{n+1-k}} \times$ $S_{\sqrt{2(k-1)}}^{k}$. Consider the function $u$ on $M$ given by

$$
u=|x|^{2}-2(n+1-k) .
$$

Then $u$ is non-negative. By Lemma 3 the function $u$ and its gradient are in $L_{f}^{2}(M)$ and satisfies

$$
0 \leq u \leq-\Delta_{f} u .
$$

As before $u$ is identically zero and $M$ is the product $S_{\sqrt{2(n+1-k)}}^{n-k} \sqrt{\sqrt{2(k-1)}}$. By Lemma 8 this product is not a self-shrinker, a contradiction.

Suppose that the self-shrinker $M$ lies in the product of the closed halfspace satisfying $\sum_{i=1}^{n+1-k} x_{i} v_{i} \geq 0$ in $\mathbb{R}^{n+1-k}$ with the sphere $S_{\sqrt{2(k-1)}}^{k}$ for some vector $v$ in $\mathbb{R}^{n+1-k}$. Consider the function $u$ on $M$ given by

$$
u=\sum_{i=1}^{n+1-k} x_{i} v_{i} .
$$

Then $u$ is non-negative. By Lemma 3 this function is a $L_{f}^{2}(M)$ eigenfunction of the drifted Laplacian of $M$ with nonzero eigenvalue. Since $M$ has finite 
weighed volume (by Lemma 1) it follows that $u$ is orthogonal to the constant function with respect to the $L_{f}^{2}(M)$ inner product i.e. $\int_{M} u e^{-f}=0$. Thus $u$ is identically zero and $M$ is the product of the hyperplane $\sum_{i=1}^{n+1-k} x_{i} v_{i}=0$ with the sphere $S_{\sqrt{2(k-1)}}^{k}$.

\section{REFERENCES}

[1] Marcos P. Cavalcante and José M. Espinar, Halfspace type theorems for self-shrinkers, Bull. Lond. Math. Soc. 48 (2016), no. 2, 242-250, DOI 10.1112/blms/bdv099. MR3483061

[2] Bing-Long Chen, Strong uniqueness of the Ricci flow, J. Differential Geom. 82 (2009), no. 2, 363-382. MR2520796

[3] Xu Cheng, Tito Mejia, and Detang Zhou, Simons-type equation for $f$-minimal hypersurfaces and applications, J. Geom. Anal. 25 (2015), no. 4, 2667-2686. MR3427142

[4] Stability and compactness for complete $f$-minimal surfaces, Trans. Amer. Math. Soc. 367 (2015), no. 6, 4041-4059, DOI 10.1090/S0002-9947-2015-06207-2. MR3324919

[5] Eigenvalue estimate and compactness for closed $f$-minimal surfaces, Pacific J. Math. 271 (2014), no. 2, 347-367, DOI 10.2140/pjm.2014.271.347. MR3267533

[6] Xu Cheng and Detang Zhou, Stability properties and gap theorem for complete fminimal hypersurfaces, Bull. Braz. Math. Soc. (N.S.) 46 (2015), no. 2, 251-274, DOI 10.1007/s00574-015-0092-z. MR3448944

[7] - Volume estimate about shrinkers, Proc. Amer. Math. Soc. 141 (2013), no. 2, 687-696, DOI 10.1090/S0002-9939-2012-11922-7. MR2996973

[8] _ Eigenvalues of the drifted Laplacian on complete metric measure spaces, Communications in Contemporary Mathematics, DOI 10.1142/S0219199716500012, (to appear in print).

[9] Huai-Dong Cao and Detang Zhou, On complete gradient shrinking Ricci solitons, J. Differential Geom. 85 (2010), no. 2, 175-185. MR2732975

[10] Tobias H. Colding and William P. Minicozzi II, Generic mean curvature flow I: generic singularities, Ann. of Math. (2) 175 (2012), no. 2, 755-833, DOI 10.4007/annals.2012.175.2.7. MR2993752

[11] Tobias Holck Colding and William P. Minicozzi II, Uniqueness of blowups and Eojasiewicz inequalities, Ann. of Math. (2) 182 (2015), no. 1, 221-285, DOI 10.4007/annals.2015.182.1.5. MR3374960

[12] Q. Ding, Y.L. Xin, and L. Yang, The rigidity theorems of self shrinkers via Gauss maps, arXiv 1203.1096.

[13] Klaus Ecker, Logarithmic Sobolev inequalities on submanifolds of Euclidean space, J. Reine Angew. Math. 522 (2000), 105-118, DOI 10.1515/crll.2000.033. MR1758578

[14] Joerg Enders, Reto Müller, and Peter M. Topping, On type-I singularities in Ricci flow, Comm. Anal. Geom. 19 (2011), no. 5, 905-922, DOI 10.4310/CAG.2011.v19.n5.a4. MR2886712

[15] M. Fernández-López and E García-Río, Rigidity of shrinking Ricci solitons, Math. Z. 269 (2011), no. 1-2, 461-466, DOI 10.1007/s00209-010-0745-y. MR2836079

[16] Richard S. Hamilton, The formation of singularities in the Ricci flow, Surveys in differential geometry, Vol. II (Cambridge, MA, 1993), Int. Press, Cambridge, MA, 1995, pp. 7-136. MR1375255

[17] D. Hoffman and W. H. Meeks III, The strong halfspace theorem for minimal surfaces, Invent. Math. 101 (1990), no. 2, 373-377, DOI 10.1007/BF01231506. MR1062966

[18] Gerhard Huisken, Deforming hypersurfaces of the sphere by their mean curvature, Math. Z. 195 (1987), no. 2, 205-219, DOI 10.1007/BF01166458. MR892052 
[19] _ Asymptotic behavior for singularities of the mean curvature flow, J. Differential Geom. 31 (1990), no. 1, 285-299. MR1030675

[20] Stephen Kleene and Niels Martin Møller, Self-shrinkers with a rotational symmetry, Trans. Amer. Math. Soc. 366 (2014), no. 8, 3943-3963, DOI 10.1090/S0002-99472014-05721-8. MR3206448

[21] Peter Li, Geometric analysis, Cambridge Studies in Advanced Mathematics, vol. 134, Cambridge University Press, Cambridge, 2012. MR2962229

[22] O. Munteanu and N. Sesum, On gradient Ricci solitons, J. Geom. Anal. 23 (2013), no. 2, 539-561, DOI 10.1007/s12220-011-9252-6. MR3023848

[23] G. Perelman, The entropy formula for the Ricci flow and its geometric applications, arXiv 0211159 .

[24] Michael Reed and Barry Simon, Methods of modern mathematical physics. IV. Analysis of operators, Academic Press [Harcourt Brace Jovanovich, Publishers], New YorkLondon, 1978. MR0493421

[25] W. Sheng and H. Yu, Evolving hypersurfaces by their mean curvature in the background manifold evolving by Ricci flow, arXiv 1407.5195.

[26] Luen-Fai Tam and Detang Zhou, Stability properties for the higher dimensional catenoid in $\mathbb{R}^{n+1}$, Proc. Amer. Math. Soc. 137 (2009), no. 10, 3451-3461, DOI 10.1090/S0002-9939-09-09962-6. MR2515414

[27] Y. Wei, On lower volume growth estimate for $f$-minimal submanifolds in gradient shrinking soliton, International Mathematics Research Notices (accepted).

[28] H. Yamamoto, Ricci-mean curvature flows in gradient shrinking Ricci solitons, arXiv 1501.06256 .

Departamento de Matemática, Universidade Federal do Espírito Santo, VitóRIA, ES 29075-910, BRAZIL.

E-mail address: matheus.vieira@ufes.br

Instituto de Matemática e Estatística, Universidade Federal Fluminense, Niterói, RJ 24020-140, BRAzIL.

E-mail address: zhou@impa.br 